\section{Endoscopic Findings in Pseudoxanthoma Elasticum}

Pseudoxanthoma elasticum is a rare, hereditary disorder of the connective tissue, characterized by degeneration of elastic fibers, and it is associated with numerous systemic complications. Clinically, it affects mainly the skin, retina, blood vessels, and myocardium. Other congenital cardiovascular, gastrointestinal, and genitourinary defects may also occur with the syndrome. In the female patient presented here, the diagnosis of pseudoxanthoma elasticum of the skin (Figure 1) was confirmed histopathologically at the age of 15 (Figure 2). Two years later, recurrent gastrointestinal bleeding occurred. At the time of admission due to bleeding, fiber-optic endoscopy revealed typical vascular changes associated with the underlying disease, such as dilated and tortuous veins in the esophagus, fundus, and duodenal bulb. An area of fresh bleeding was found just below the esophagogastric junction (Figure 3). After endoscopic injection of adrenalin (1:10 000) into the bleeding areas, hemorrhage stopped. During the following months, the patient was frequently admitted to the hospital with bleeding episodes from esophageal varices, which were successfully treated by endoscopic sclerotherapy. Subsequently she recovered and remained asymptomatic for a further eight months.

Gastrointestinal hemorrhage ranks as one of the most frequent and troublesome complications of pseudoxanthoma elasticum, occurring in $14 \%$ of patients in one series (1). Endoscopic findings include a distinctive yellow cobblestone appearance of the mucosa, which correlates histologically to dilated mucosal and submucosal veins and capillaries, along with fragmentation and calcification of the internal elastic lamina and intimal sclerosis of the small and medium-sized arteries (2). These vascular alterations may lead to persistent bleeding due to a failure of vascular constriction. In view of the underlying connective-tissue disease, endoscopic hemostasis should be regarded as preferable to surgery.

\section{H. Fruhwirth ${ }^{l}$, H. Rabl ${ }^{l}$, H. Hauser ${ }^{l}$, C. Schmid ${ }^{2}$, A. Beham ${ }^{2}$,} G. E. Klein ${ }^{3}$

Department of General Surgery, ${ }^{2}$ Department of Pathology, and

${ }^{3}$ Department of Radiology, University School of Medicine, Graz. Austria

\section{References}

1 Kaplan L, Hartmann SW: Elastica disease: case of GrönbladStrandberg syndrome with gastrointestinal hemorrhage. Arch. Intern. Med. 1954; 84: 484-492.

2 Kundrotas L Novak J, Kremzier J, et al.: Gastric bleeding in pseudoxanthoma elasticum. Am. J. Gastroenterol. 1988; 83: 868-874.

Corresponding Author

H. Fruhwirth, M.D.

Department of General Surgery

Karl-Franzens-Universität Graz

Auenbruggerplatz 29

8036 Graz, Austria

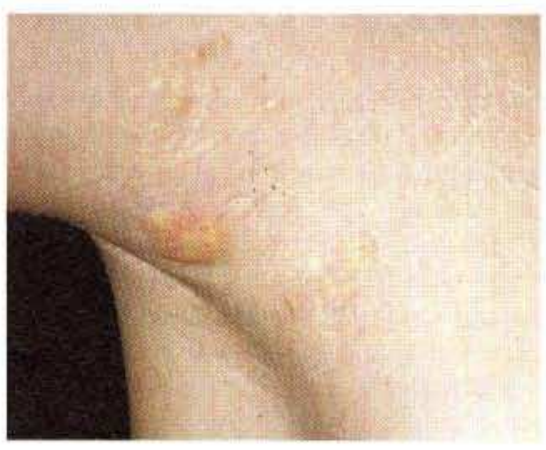

Figure 1:

Areas of yellowish

skin with a goose-

flesh appearance

on the upper

chest and axilla

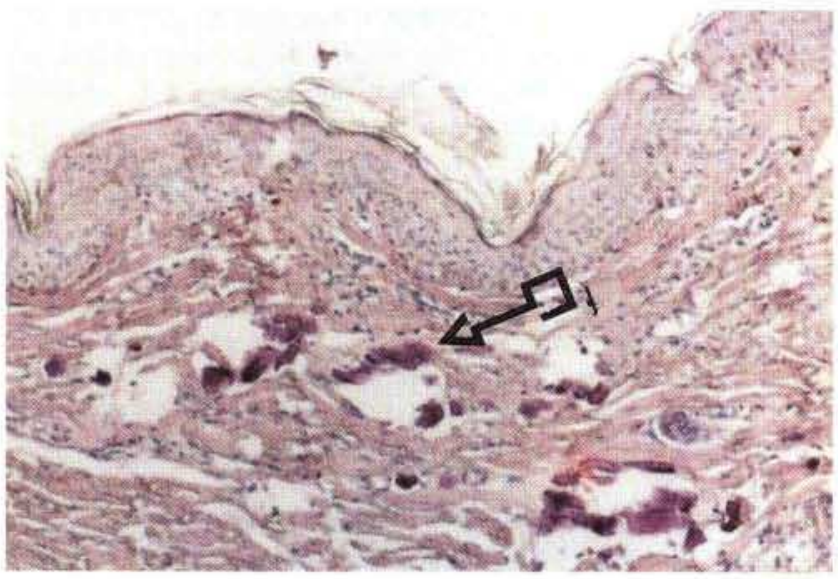

Figure 2: Histology of the skin arteries, showing degenerative changes and high-grade calcification, especially in the internal elastic lamella and in the media (arrow) $(\mathrm{HE} \times 60)$.

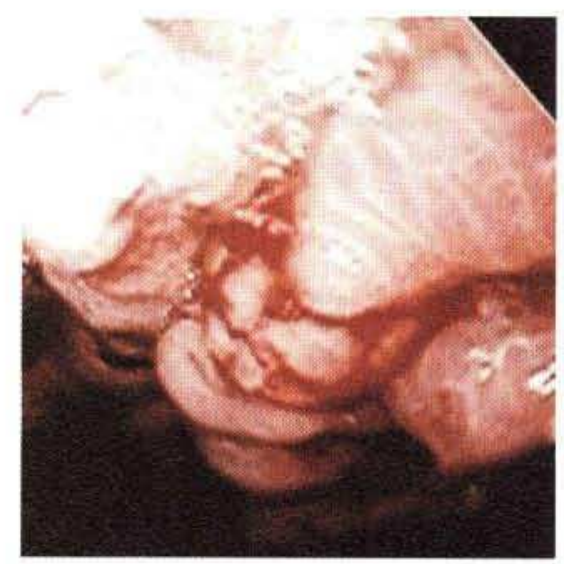

Figure 3:

Esophagogastroscopy showing highly dilated submucosal veins protruding into the lumen of the esophagus, with a fresh bleeding area. 